American Journal of Applied Sciences 4 (11): 896-901, 2007

ISSN 1546-9239

(C) 2007 Science Publications

\title{
A 4×1 L-probe fed Inverted Hybrid E-H Microstrip Patch Antenna Array for 3G Application
}

\author{
${ }^{1}$ Mohammad Tariqul Islam, ${ }^{2}$ Norbahiah Misran, and ${ }^{2} \mathrm{Ng}$ Kok Jiunn \\ ${ }^{1}$ Faculty of Engineering and Technology, Multimedia University, 75450 Melaka, Malaysia \\ ${ }^{2}$ Department of Electrical, Electronics and System Engineering, \\ University Kebangsaan Malaysia, Bangi, Malaysia
}

\begin{abstract}
This paper presents a compact and broadband microstrip patch antenna array (BMPAA) with uniform linear array configuration of $4 \times 1$ element for $3 \mathrm{G}$ applications. The $4 \times 1$ BMPAA was designed and developed by integrating new patch shape Hybrid E-H shape, L-probe feeding, inverted patch structure with air filled dielectric microstrip patch antenna (MPA) element. The array was constructed using two dielectric layer arrangement with a thick air-filled substrate sandwiched between a top-loaded dielectric substrate ( $R T$ 5880) with inverting radiating patch and a ground plane. The Lprobe fed inverted hybrid E-H (LIEH) shaped BMPAA achieves an impedance bandwidth of $17.32 \%$ referenced to the center frequency at $2.02 \mathrm{GHz}$ (at VSWR $\leq 1.5$ ), maximum achievable gain of $11.9 \pm 1 \mathrm{dBi}$, and $23 \mathrm{~dB}$ crosspolarization level.
\end{abstract}

Key words: 4X1 Array, BMPAA, MPA, hybrid E-H shape patch; L-probe fed

\section{INTRODUCTION}

The demand for mobile communication services is increasing at a rapid pace throughout the globe. The total number of subscribers worldwide is projected to increase from 283 million in 2002 to approximately 1.2 billion in 2007. In addition, multimedia traffic is also projected to increase 40 percent every year, especially in Asia. The International Telecommunication Union (ITU) forecasted that the amount of wireless multimedia traffic in 2010 will be 5.4 times larger than the amount recorded in 1999 due to the provisioning of high-speed data services ${ }^{[1]}$. Science the demand for high data rate services is increasing, a more efficient use of the radio spectrum is required. To meet these requirements, ITU proposed the 3G/IMT-2000 system, formerly known as future public land mobile telecommunications service (FPLMTS). The 3G system covers the operating frequency range of 1.885 to $2.025 \mathrm{GHz}$ for uplink and range of 2.110 to $2.200 \mathrm{GHz}$ for downlink.

One of the main focuses of $3 \mathrm{G}$ is to develop an antenna system that can direct the antenna beam to the intended users while placing a null to the interferers in a dynamic fashion, the architecture of smart antenna system. The application of smart antenna for 3G networks will revolutionize at least the base station antenna design. As the number of mobile operator increases, the requirements to support better mobile services in a limited radio environment become a major challenge. Hence, a solution whereby a common antenna shared by various operators are needed, this will also reduce the un-aesthetically or unpleasing base station sites. One of the main challenges for the base station antenna design is to come up with an antenna system with smart antenna capabilities covering 3G bandwidth and with a low-profile structure for easy installation and maintenance. In an antenna array multiple radiating elements are assembled in an electrical and geometrical configuration. The antenna array provides beamforming capability to extract the desired signal and simultaneously filtering out the unwanted interference signal and environmental noise $^{[2]}$.

In designing array antenna, dipole, horn, waveguide slot or microstrip antenna can be used as radiating elements. However such radiating elements are unable to meet the requirements to be aesthetic and light weighted if implemented on the cell sites. To be

Corresponding Author: $\quad$ Mohammad Tariqul Islam, Faculty of Engineering and Technology, Multimedia University, Jalan Ayer Keroh Lama, 75450 Melaka, Malaysia, Tel: +606-2523023, Fax: +606-2316552 
aesthetic, the radiating element of the antenna array is desired to be small in size, low profile, conformal and compatible with integrated circuit and etc. Therefore, the radiating elements are preferred to be fabricated by microstrip technology that offers its non-electrical characteristic advantages. Microstrip antennas are low profile and light weight. They can also be made conformable and well suited to be integrated with microwave integrated circuit ${ }^{[3,4]}$. Most of the current researches on MPAs have been devoted on broadband techniques ${ }^{[5-8]}$, printed array and feeding techniques ${ }^{[9-10]}$ to meet the current broadband applications. The following section discusses the design of LIEH shaped MPA, development of $4 \times 1$ BMPAA and measurement results.

\section{LIEH SHAPED MPA DESIGN}

The basic geometry of the LIEH antenna element is shown in Fig. 1. The inverted rectangular patch, with width $W$ and length $L$ is supported by a low dielectric superstrate with dielectric permittivity $\varepsilon_{r 1}$ and thickness $h_{1}$. An air-filled substrate with dielectric permittivity $\varepsilon_{o}$ and thickness $h_{o}$ is sandwiched between the superstrate and a ground plane. The LIEH patch antenna integrates both the $\mathrm{E}$ - and $\mathrm{H}$-shaped patch on the same radiating element. For the E-shaped, the slots are embedded in parallel on the radiating edge of the patch, symmetrically with respect to the centreline ( $\mathrm{x}$ - axis) of the patch; while for the $\mathrm{H}$-shaped the slots are embedded in serial on the non-radiating edge of the patch, symmetrically with respect to the centreline.

The design adopted the two-dielectric layers approach $^{[10]}$ with a low permittivity dielectric forming the superstrate supporting the inverted radiating element above a ground plane with an air-filled substrate sandwiched between them. The E- and $\mathrm{H}-$ shaped slots $\left\{l_{E}, w_{E}\right.$, and $\left.s_{E}\right\}$ and $\left\{l_{H}, w_{H}\right.$ and $\left.s_{H}\right\}$ are shown in Fig. 1 (a), where, $l, w$, and $s$ are the length, width, and position of the slots. The patch is fed by an L-shaped probe with width $\left(w_{p}\right)$ of $1 \mathrm{~mm}$, height, $h_{P}$ and horizontal length, $l_{P}$ along the centerline (x-axis) at a distance $f_{P}$ from the edge of the patch as shown in Fig. 1(b).

The novel LIEH patch antenna design reduces the sizes to $79 \mathrm{~mm} \times 41 \mathrm{~mm}$, which is about $22.64 \%$ reduction, compared with the inverted E-shaped rectangular microstrip patch antenna designed by ${ }^{[10]}$. An L-shaped probe is employed for feeding the patch element. This L-shaped probe is an excellent feeding technique for a thick substrate (thickness $\approx 0.1 \lambda$ ) microstrip antenna, where the high inductance of the long pin probe can be suppressed by the capacitance which is introduced by the bend of the probe itself. This feeding technique also offers easy fabrication where soldering to the patch itself can be avoided and the probe can be bent easily. These advantages are very favorable especially in an array structure.

The L-probe feeding technique with a thick airfilled substrate has been reported to enhance the antenna bandwidth ${ }^{[11,12,13]}$, while inverted radiating patch offers a gain enhancement ${ }^{[2]}$ and at the same time protects from the environmental effect. Both techniques offer easy patch fabrications, especially for array structures. The use of parallel slots on the patch ${ }^{[12]}$, on the other hand, improves cross polarization while the introduction of series slots reduces the size of the patch $^{[14,15]}$. In addition, by employing the air-filled substrate, the substrate thickness can be adjusted easily in the design by adjusting the spacer and it is more cost effective, eliminating the deformity problem of the foam material. Further the design facilitates the fabrication of the patch using the printed circuit board (PCB) technique.

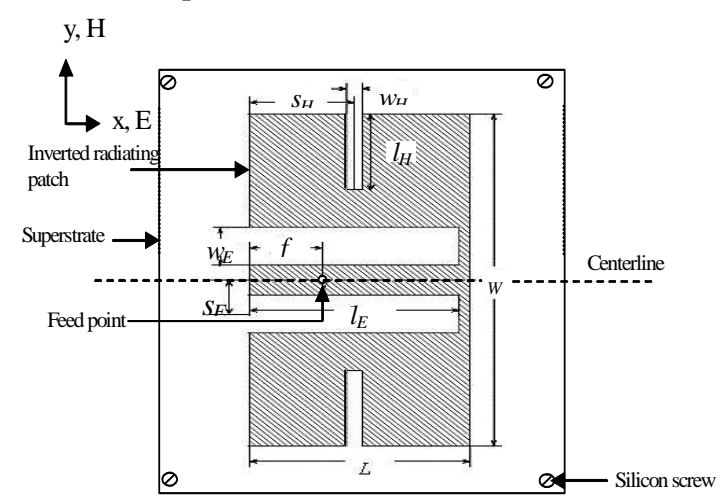

(a)

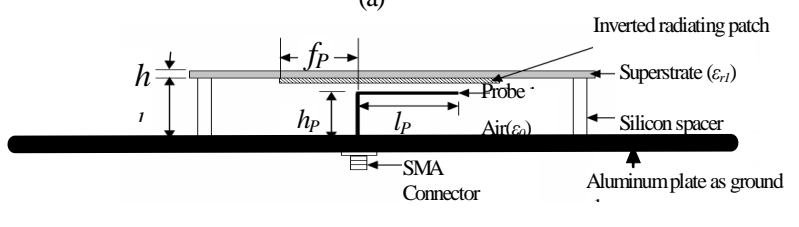

(b)

Fig 1 (a) Top view and (b)side view of LIEH shaped MPA 


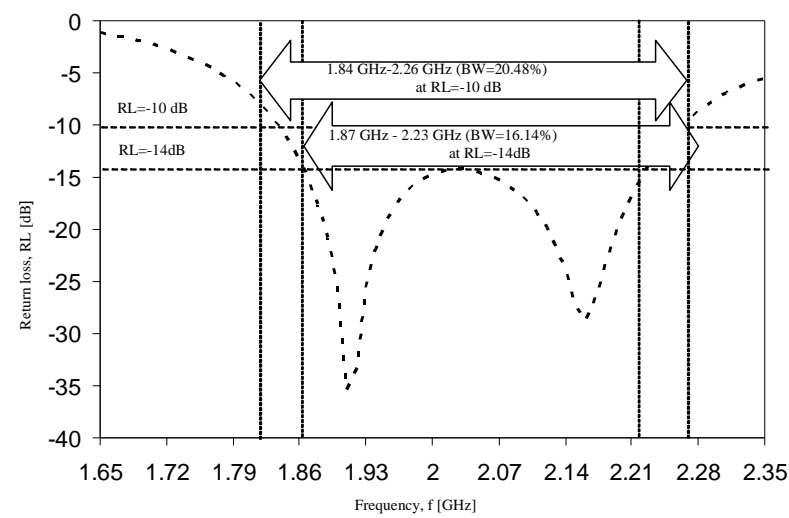

Fig. 2: Simulated return loss of the LIEH shaped MPA

\section{SIMULATION RESULTS}

A commercial electromagnetic simulator Sonnet ${ }^{\circledR}$ Suite Version is used to obtain the optimized design parameters. The simulation results of the impedance bandwidth and gain obtained from the LIEH shaped MPA element is shown Fig. 2 and 3. Fig. 2 gives the simulated return loss (RL) of the LIEH shaped single MPA element.

Noted in this figure, the antenna resonates at 1.91 $\mathrm{GHz}$ and at $2.16 \mathrm{GHz}$ where these closely excited adjacent resonant frequencies resulted in a broadband characteristic of the antenna. These dual resonance frequencies are due to the effect introduced by the embedded parallel slots structure of the rectangular patch. The bandwidth can be calculated from the RL plot. The bandwidth of the antenna can be said to be those range of frequencies over which the RL is greater than $-10 \mathrm{~dB}$ corresponds to a VSWR of 2 which is acceptable. As shown in Fig. 2, the simulated impedance bandwidth is about $20.48 \%$, covering the frequency range from 1.84-2.26 GHz. These impedance bandwidth values are determined at return loss level of $10 \mathrm{~dB}$ or at VSWR $=2$. An achievable bandwidth of $16.14 \%$ is obtained, covering the frequency range from $1.87-2.23 \mathrm{GHz}$ at $14 \mathrm{~dB}$ return loss or at VSWR 1.5.

The simulated gain of the LIEH shaped patch antenna at various frequencies is shown in Fig. 3. The maximum achievable gain is $8.31 \mathrm{~dB}$ and the gain variation is about $1.39 \mathrm{dBi}$ between the frequency ranges of $1.85 \mathrm{GHz}$ to $2.2 \mathrm{GHz}$ (i.e. a gain bandwidth of $15.90 \%$ ) which is inside the $3 \mathrm{~dB}$ gain band.

The array has employed the LIEH shaped patch with the dimension of each element $\{W, L\}=\{79,41\}$ $\mathrm{mm}$ and inter-element spacing of $68 \mathrm{~mm}$ (or $0.50 \lambda$ ) at $2.2 \mathrm{GHz}$. The total dimension of the array is $120 \mathrm{~mm}$ (width) $\times 285 \mathrm{~mm}$ (length) with the size of the ground plane is $370 \mathrm{~mm} \times 200 \mathrm{~mm} \times 1 \mathrm{~mm}$.

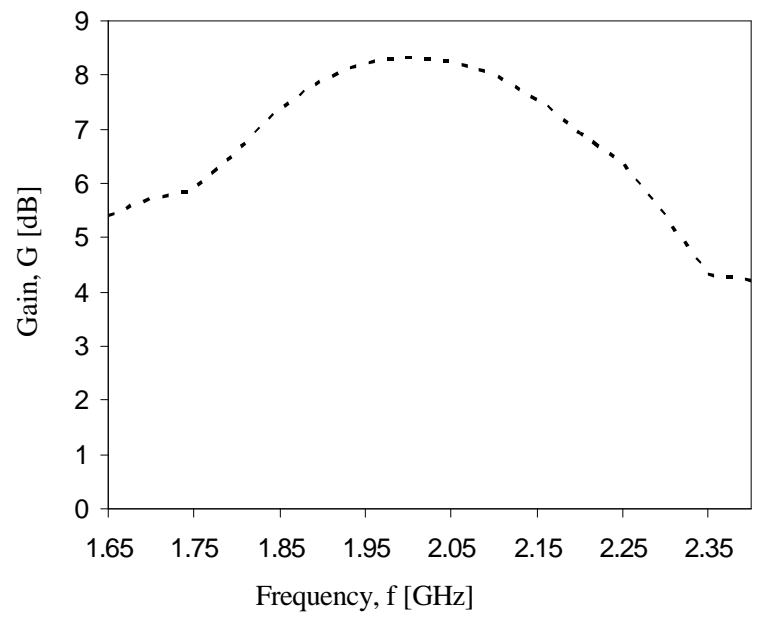

Fig. 4: (a) Top view, (b) side view of the $4 \times 1 \mathrm{LIEH}$ patch elements

The fabricated $4 \times 1$ linear antenna array is shown in Fig. 5. The antenna array is fabricated on Rogers RT 5880 with dielectric permittivity, $\varepsilon_{r 1}$ of 2.2 and thickness, $h_{1}$ of $1.5748 \mathrm{~mm}$. The thickness of the airfilled substrate, $h_{0}$ is $16 \mathrm{~mm}$. The array antenna employs four identical L-shaped probe feds of copper wire with a radius of $1 \mathrm{~mm}$ as shown in Fig. 4(b). The fabricated $4 \times 1$ inverted hybrid E-H shaped patch and the ground plane are assembled together using silicon spacers and screws ( $3 \mathrm{~mm}$ diameter).

The horizontal and vertical dimensions of each probe are $25 \mathrm{~mm}$ and $14 \mathrm{~mm}$, respectively. All the Lshaped probes are connected to the feed network which is located underneath the ground plane via SMA connectors.

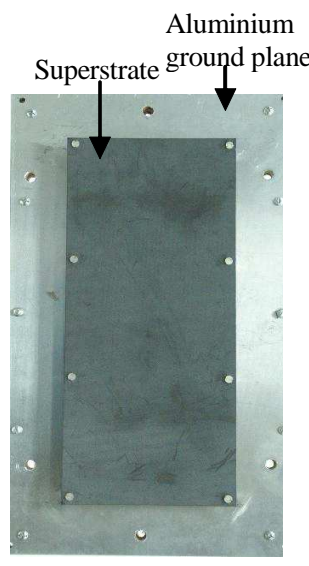

(a)

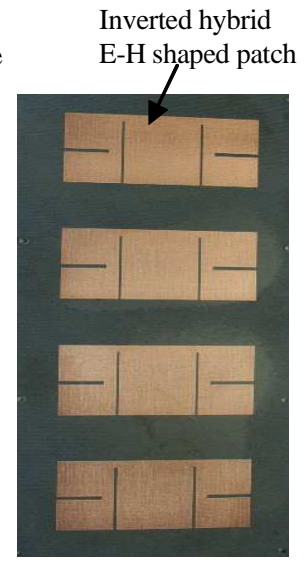

(b)
Fig. 5: The fabricated $4 \times 1$ LIEH shaped BMPAA: (a) top view, (b) inside view showing inverted element 


\section{RESULTS AND DISCUSSION}

The LIEH MPA element and the $4 \times 1$ BMPAA are simulated by Sonnet ${ }^{\circledast}$ Suite em simulator. The fabricated antennas are measured using the Agilent PNA E8358A network analyzer, Agilent ESG-DP Series E4436B signal generator, Advantest R3131A spectrum analyzer and the standard gain LPDA-0803 log periodic dipole antenna. SMA connector calibration kit, SMA male to male cable and automatic controlled rotator are also required for the measurement. Measurement is conducted in the UKM microwave lab and the UKM open field.

Fig. 6 shows the measured RL for the four elements. From the Fig. 6, it can be observed that the RL shows almost similar pattern for the all four elements. Simulation results agree well to the measured results but are not presented for the sake of brevity. The $\mathrm{RL}$ of four elements shows two distinct resonance frequencies at 1.91 and $2.14 \mathrm{GHz}$ as shown in the figure. These closely excited adjacent resonant frequencies are contributed to the wideband characteristic of the antenna array. The VSWR is less than 2 in the frequency range from 1.80 to $2.24 \mathrm{GHz}$ equivalent to an impedance bandwidth of $21.78 \%$. At VSWR less than 1.5, the impedance bandwidth is $17.32 \%$ for the frequency range between 1.855 and 2.20 $\mathrm{GHz}$, which is wide enough to cover the $3 \mathrm{G}$ or IMT2000 band.

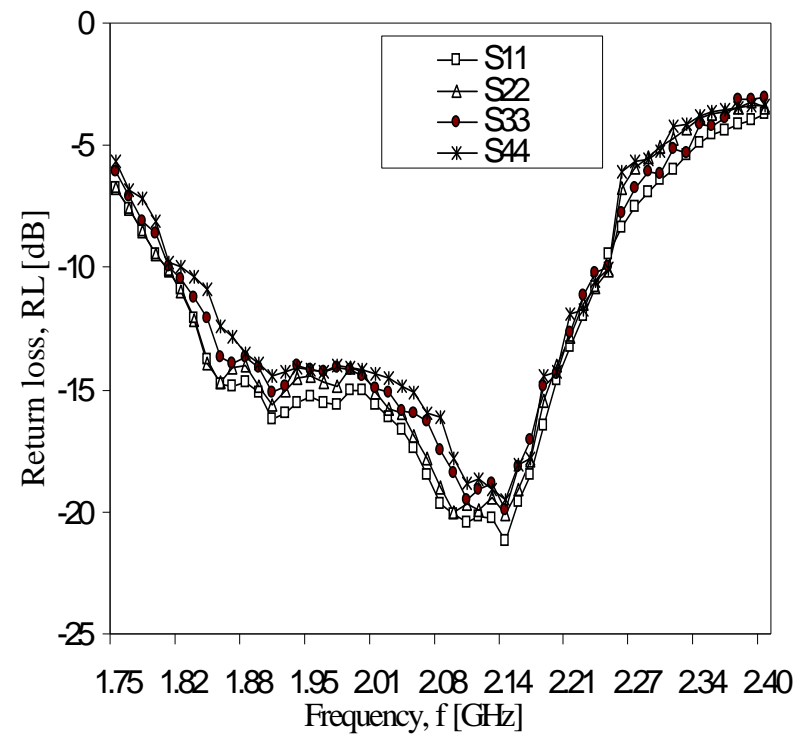

Fig. 6: Return loss for $4 \times 1$ LIEH shaped BMPAA
Figs. 7 and 8 show the E-plane and H-plane radiation patterns of the antenna array at $1.91 \mathrm{GHz}$ and $2.14 \mathrm{GHz}$ (resonance frequencies). Chebyshev amplitude distribution is used to have narrow beamwidth and low equal side lobe level $(-20 \mathrm{~dB})$. The radiation patterns show some fluctuations due to the reflection from some obstacles in the field, however, they have good beam patterns and crosspolarization level, which is shown in Figs. 7 and 8. The $3 \mathrm{~dB}$ beamwidth is closed to $25^{\circ}$ for the E-plane while the 3 $\mathrm{dB}$ beamwidth is about $65^{\circ}$ for the H-plane. The Hplane radiation pattern is virtually symmetrical, while the E-plane radiation pattern exhibits some asymmetries.

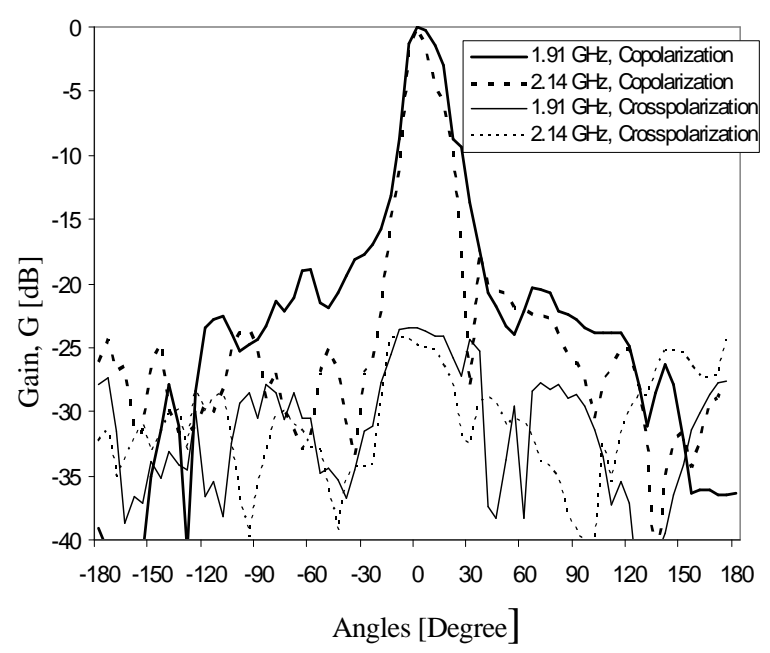

Fig. 7: Measured E-plane radiation patterns for the LIEH shaped BMPAA at $1.91 \mathrm{GHz}$ and 2.14 $\mathrm{GHz}$

As shown in Fig. 7, the sidelobe levels are unequally distributed. The first side lobe levels at 1.91 $\mathrm{GHz}$ and $2.14 \mathrm{GHz}$ are $-18.9 \mathrm{~dB}$ (at $-60^{\circ}$ ) and $-20.47 \mathrm{~dB}$ (at $45^{\circ}$ ) respectively. This result is due to the amplitude/phase unbalances in the beamforming feed network. The maximum crosspolarization of the array is in the order of $-23.47 \mathrm{~dB}$ and $-10 \mathrm{~dB}$ for E-plane and $\mathrm{H}$ plane respectively. 


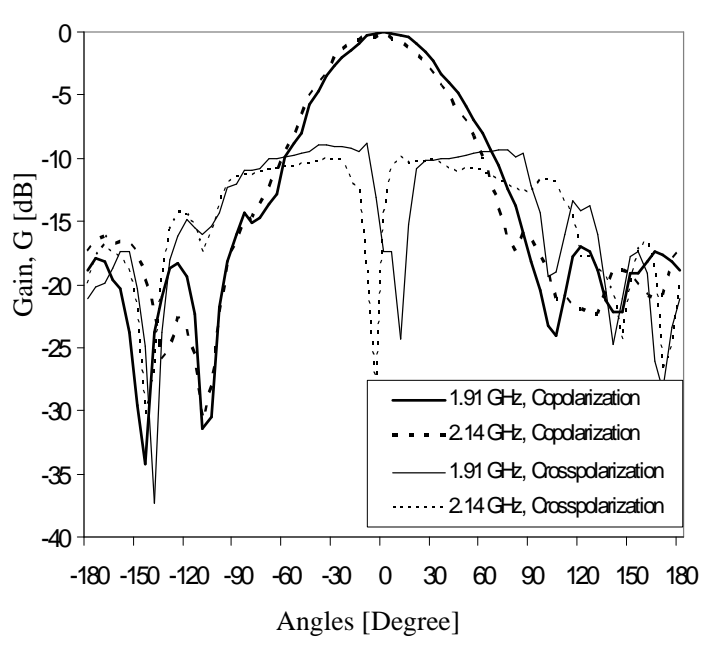

Fig. 8: Measured H-plane radiation patterns for the LIEH shaped BMPAA at $1.91 \mathrm{GHz}$ and 2.14 $\mathrm{GHz}$

The measured gain of the LIEH shaped patch antenna at various frequencies is shown in Fig. 9.

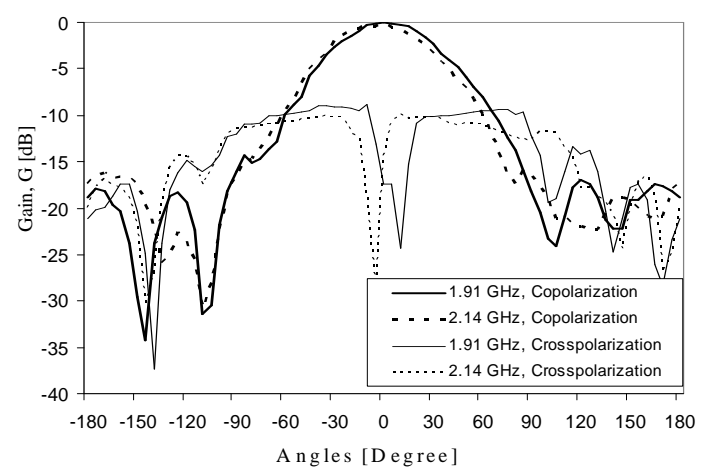

Fig. 9: Measured gain of LIEH shaped BMPAA at different frequencies

As shown in the Fig., the maximum achievable gain is $11.9 \mathrm{~dB}$ with $1.28 \mathrm{~dB}$ gain variation between the frequency ranges of $1.85 \mathrm{GHz}$ to $2.2 \mathrm{GHz}$ (i.e. a gain bandwidth of $15.90 \%$ ) which is inside the $3 \mathrm{~dB}$ gain band.

\section{CONCLUSION}

Due to the high cost and complexity of the design for planar and high resolution array, the design focused on the development of a uniform four-element $(4 \times 1)$ array antenna. The array designed by employing novel hybrid E-H shaped design, inverted patch, slotted patch and L-probe, which has provided better size reduction (22\% reduction compared to the normal rectangular patch) and crosspolarization apart from the broadband features of the patch. The BMPAA with the elements spacing of $0.5 \lambda$, provides an impedance bandwidth of $440 \mathrm{MHz}$ or about $21.78 \%$ at $10 \mathrm{~dB}$ return loss, referenced to the center frequency of $2.02 \mathrm{GHz}$. The crosspolarization radiation, in the E-plane patterns of the array is about $-23 \mathrm{~dB}$ and $-10 \mathrm{~dB}$ for the H-plane. The LIEH patch has a compact dimension of $79 \times 41$ $\mathrm{mm}^{2}$.

\section{ACKNOWLEDGEMENTS}

The authors would like to thank the IRPA Secretariat, Ministry of Science, Technology and Environmental of Malaysia, IRPA Grant: 04-02-020029 and Institute for Space Scienec, UKM, UKM Grant: LL-001-2004 for sponsoring this work.

\section{REFERENCES}

1. Ryu, S., D. Oh, G. Sihn, K. Han, and S. Hwang, 2005. Research activities on next- generation mobile communications and services in Korea. IEEE Communications Magazine, 36(9): 122-131.

2. Ng, K.J., Z.A. Abdul Rashid, M.T. Islam, 2003. Broadband inverted E-shaped rectangular microstrip patch antennas for 3G applications. IEEE National Symposium on Microelectronics, NSM-2003, Perlis, Malaysia, 286-289.

3. Lau, K.-L., K.-M. Luk, and K.-F. Lee, 2006. Design of a circularly-polarized vertical patch antenna. IEEE Transactions on Antennas and Propagation, 54(4): 13321335.

4. Zhang, Y.P. and J.J. Wang, 2006. Theory and analysis of differentially-driven microstrip antennas. IEEE Transactions on Antennas and Propagation, 54(4): 10921099.

5. Huynh, T. and K.F. Lee, 1995, Single-layer single-patch wideband microstrip antenna. Electronics Letters 31(16): 1310-1312.

6. Lee, K.F., K.M. Luk, K.F.Tong, S.M., Shum, T. Hunh, and R.Q. Lee, 1997. Experimental and simulation studies of the coaxially fed U-slot rectangular patch antenna. IEE Proceedings on Microwaves, Antennas and Propagation, 144: 354-358.

7. Wong, K.L and W.H. Hsu, 2001. A broad-band rectangular patch antenna with a pair wide slits. IEEE Transactions on Antennas Propagation 49(9): 1345-1347.

8. Yang, F., X. Zhang, and Y. Rahmat-Samii, 2001. Wideband E-shaped patch antennas for wireless communications. IEEE Transactions on Antennas and Propagation, 49(7): 1094-1100. 
9. An, Y., L. Xin, and G. Benqing, 2000. Developing a kind of microstrip array antenna with beam squint. Proceedings of 5th International Symposium on Antennas, Propagation and EM Theory, ISAPE 2000, IEEE, pp. 443-446.

10. Jeong, K. II and Y.J. Yoon, 2000. Design of wideband microstrip array antennas using the coupled lines. IEEE International Symposium on Antennas and Propagation Society 3: 410-1413.

11. Guo, Y-X. and C.L. Mak, 2001. L-Probe proximity-fed annular ring microstrip antennas. IEEE Transactions on Antennas and Propagation, 49(1): 19-21.

12. Mak, C.L., K.F. Lee and K.M. Luk, 1999. Microstrip line-fed L-strip patch antenna. IEEE Proceedings on Microwaves, Antennas and Propagation, 146(4): 282284.
13. Mak, C.L. and K.M. Luk, 2000. Experimental study of a microstrip patch antenna with an L-shaped probe. IEEE Transactions on Antennas and Propagation, 48(5): 77-78.

14. Hong, C.S. 1999. Gain-enhanced broadband microstrip antenna. Proceedings of the National Science Council, Republic of China, ROC(A), 23(5): 609-611.

15. Mahmoud, S.F. and A.F. Sheta, 2001. A novel H-shaped patch antenna. International Symposium on Antennas and Propagation Society, 2: 720-723. 